\title{
ON A CYCLIC SUM
}

\author{
by P. H. DIANANDA
}

(Received 2 December, 1961)

1. For any positive integral $n$ and any positive real $x_{1}, \ldots, x_{n}$ we write

$$
S_{n}\left(x_{1}, \ldots, x_{n}\right)=\sum_{r=1}^{n} \frac{x_{r}}{x_{r+1}+x_{r+2}},
$$

where

$$
x_{n+r}=x_{r} \quad(\text { all } r) .
$$

Let

$$
\lambda(n)=(1 / n) \inf _{x_{1}, \ldots, x_{n}} S_{n}\left(x_{1}, \ldots, x_{n}\right) \text {. }
$$

Then

$$
\lambda(n) \leqq \frac{1}{2}
$$

clearly. It is known $[1,2]$ that

$$
\lambda(n)=\frac{1}{2}
$$

for $n \leqq 6$, and further $[4,5,6]$ that (5) is false for even $n \geqq 14$ and for odd $n \geqq 53$. Mordell [2] conjectured that (5) is false for all $n \geqq 7$, but recently [3] stated that computations indicated that (5) is true for $n=7$ and gave some calculations in support of (5) for $n=7$.

In this note we shall prove the

THEOREM. If (5) is false for $n=m$, where $m$ is odd, then (5) is false for all $n \geqq m$.

An immediate consequence of this theorem and equality (7) given later is the

COROLlaRY. If (5) is true for $n=m$, where $m$ is even, then (5) is true for all $n \leqq m$.

In the preceding paper (as Professor R. A. Rankin has kindly informed me) Djoković has proved that (5) is true for $n=8$. A consequence of this result and the corollary is that (5) is true for all $n \leqq 8$ and, in particular, for $n=7$. This confirms the truth of the result indicated by computations and referred to earlier.

2. To prove the theorem we note first, from (3) and (4), that (5) is false if and only if positive $x_{1}, \ldots, x_{n}$ exist such that

$$
S_{n}\left(x_{1}, \ldots, x_{n}\right)<\frac{1}{2} n
$$

and second, from (1) and (2), that

$$
S_{n+2}\left(x_{1}, \ldots, x_{n}, x_{1}, x_{2}\right)=S_{n}\left(x_{1}, \ldots, x_{n}\right)+1 .
$$

Hence (5) is false for $n=k+2$ if (5) is false for $n=k$. To prove the theorem it is therefore sufficient to prove the 
Lemma. If $n$ is odd, and positive $x_{1}, \ldots, x_{n}$ exist such that (6) is true, then positive $y_{1}, \ldots, y_{n+1}$ exist such that

$$
S_{n+1}\left(y_{1}, \ldots, y_{n+1}\right)<\frac{1}{2}(n+1) .
$$

To prove the lemma we note, from (1) and (2), that

$$
\begin{aligned}
S_{n+1}\left(x_{1}, \ldots, x_{r}, x_{r}, x_{r+1}, \ldots, x_{n}\right) & -S_{n}\left(x_{1}, \ldots, x_{n}\right)-\frac{1}{2} \\
= & \frac{x_{r-1}}{2 x_{r}}+\frac{x_{r}}{x_{r}+x_{r+1}}-\frac{x_{r-1}}{x_{r}+x_{r+1}}-\frac{1}{2}=\frac{\left(x_{r}-x_{r-1}\right)\left(x_{r}-x_{r+1}\right)}{2 x_{r}\left(x_{r}+x_{r+1}\right)},
\end{aligned}
$$

where

$$
x_{n+1}=x_{1} \text { and } x_{n}=x_{0} \text {. }
$$

Hence the lemma follows if, for some $r$,

$$
\left(x_{r}-x_{r-1}\right)\left(x_{r}-x_{r+1}\right) \leqq 0,
$$

where

$$
1 \leqq r \leqq n \quad(n \text { odd }), \quad x_{n+1}=x_{1}, \quad x_{n}=x_{0} .
$$

The lemma is thus proved since the assumption that (8) is false for all $r$, that is that

$$
\left(x_{r}-x_{r-1}\right)\left(x_{r}-x_{r+1}\right)>0
$$

for all $r$, leads to a contradiction. This is easily seen since, if (10) is true for all $r$, we have

and so, in virtue of (9),

$$
\prod_{r=1}^{n}\left(x_{r}-x_{r-1}\right)\left(x_{r}-x_{r+1}\right)>0
$$

$$
\prod_{r=1}^{n}\left(x_{r}-x_{r+1}\right)^{2}<0
$$

which is impossible. This concludes the proofs of the lemma and the theorem.

3. [Added, 18th December, 1961] Rankin [4] proved that the inequality (5) is false for large enough odd $n$. Later Zulauf [6] obtained the result, stated earlier, that (5) is false for odd $n \geqq 53$. We can now improve this result and prove that the inequality (5) is false for all odd $n \geqq 27$.

In what follows we let the $x_{r}$, in (1), be non-negative real numbers such that no denominator in (1) is zero. We note, from considerations of continuity, that this is permissible.

In (1), let $n=27$ and $x_{1}, \ldots, x_{27}$ be the sequence $0,7,0,8,0,9,0,10,0,11,1,12,3,11$, $5,9,6,7,6,5,6,3,6,2,6,1,6$. Then

$$
S_{27}\left(x_{1}, \ldots, x_{27}\right)=13.4990440 \ldots<27 / 2
$$

and so, by (7), our result follows.

The sequence $x_{1}, \ldots, x_{27}$ may seem to be chaotic. It is therefore interesting to note that there is some order in the sequence $x_{1}, x_{3}, \ldots, x_{53}$ or $x_{1}, x_{3}, \ldots, x_{27}, x_{2}, x_{4}, \ldots, x_{26}$ which is $0,0,0,0,0,1,3,5,6,6,6,6,6,6,7,8,9,10,11,12,11,9,7,5,3,2,1$.

In obtaining our sequence $x_{1}, \ldots, x_{27}$ we first found, by Zulauf's method (see [6]), using initially a sequence $x_{1}, \ldots, x_{24}$ for which $S_{24}\left(x_{1}, \ldots, x_{24}\right)<12$, a sequence $x_{1}, \ldots, x_{33}$ 
for which $S_{33}=S_{33}\left(x_{1}, \ldots, x_{33}\right)=33 / 2$ nearly. We then altered this sequence, a member at a time, to make $S_{33}$ as small as the order in which we altered the members enabled us to. We next deleted the pair of consecutive (in the cyclic sense) members with ratio nearest one and obtained a sequence $x_{1}, \ldots, x_{31}$ for which $S_{31}\left(x_{1}, \ldots, x_{31}\right)=31 / 2$ nearly. Using similar procedures twice more and then altering suitably the sequence obtained, we finally obtained our sequence $x_{1}, \ldots, x_{27}$. We could not obtain, by this method, a sequence $x_{1}, \ldots, x_{25}$ for which $S_{25}\left(x_{1}, \ldots, x_{25}\right)<25 / 2$. In our numerical work only integral $x_{r}$ were used.

\section{REFERENCES} 489-491.

1. P. H. Diananda, Extensions of an inequality of H. S. Shapiro, Amer. Math. Monthly 66 (1959),

2. L. J. Mordell, On the inequality $\sum_{r=1}^{n} x_{r} /\left(x_{r+1}+x_{r+2}\right) \geqq \frac{1}{2} n$ and some others, Abh. Math. Sem. Univ. Hamburg 22 (1958), 229-240.

3. L. J. Mordell, Note on the inequality $\sum_{r=1}^{n} x_{l} /\left(x_{r+1}+x_{r+2}\right) \geqq \frac{1}{2} n$, J. London Math. Soc. 37 (1962), 176-178.

4. R. A. Rankin, An inequality, Math. Gaz. 42 (1958), 39-40.

5. A. Zulauf, Note on a conjecture of L. J. Mordell, Abh. Math. Sem. Univ. Hamburg 22 (1958), 240-241.

6. A. Zulauf, On a conjecture of L. J. Mordell, II, Math. Gaz. 43 (1959), 182-184.

Department of Mathematics

UNIVERSITY OF SINGAPORE 